\title{
Kommunikationsprobleme in der medizinhistorischen Mittelalterforschung
}

\author{
Ortrun Riha
}

\section{SUMMARY}

Although medieval medical research on scientific prose has a tradition of a hundred years, its results are largely ignored by the scientific community. The reason for this is not only a shift of interest towards the social history of medicine but, more important, a deficiency in the fields of systematology and terminology which makes communication difficult, if not impossible. Most regrettable is the lack of a comprehensive review of the texts and their topics which could serve as a basis for further methodological discussion.

\section{ZuSAMMENFASSUNG}

Obwohl die landessprachige Medizinliteratur des Mittelalters gut erschlossen ist, sind die Ergebnisse dieser Fachprosaforschung praktisch unbekannt. Gründe dafür sind ein tiefgreifender Wandel in medizinhistorischer Fragestellung und Methodik, die Plazierung gleichsam in einem Leerraum zwischen Geschichte und Philologie sowie vor allem das Fehlen einer übersichtlichen Gesamtdarstellung, was wiederum an Unsicherheiten in Systematik (Abgrenzung einzelner artes, Einschätzung der Textdignität) und Terminologie liegt.

Alle bisher erschienenen - auch die neuesten - Lehrbücher der Medizingeschichte ähneln einander in Art und Tendenz ihrer Darstellung, setzen damit die von den beiden «Klassikern» der Jahrhundertwende (Walter Pagel, Max Neuburger) geprägte Tradition fort und vernachlässigen dabei praktisch durchweg die Ergebnisse der rezenten Forschung. ${ }^{1}$ Dies gilt mehr oder weniger für alle Kapitel und ist zunächst nicht weiter tragisch : es liegt an den spezifischen Erfordernissen der Gattung, die zu Kürze und Verkür- 
zung zwingen; in den Kompendien, die der Markt verlangt, bleibt kein Raum für Methoden-, Literatur- oder gar Quellendiskussion. Vermittelt werden muss abfragbares Wissen, denn die Rezipienten sind nicht Geistes-, sondern Naturwissenschaftler, die an Fakten, also an «harte Daten», gewöhnt sind und primär kein Verständnis dafür aufbringen, dass man historische «Realität» unter verschiedenen Gesichtspunkten betrachten kann. Historie erscheint dem Studenten als Abfolge von Entdeckungen und Erfindungen, die die Menschheit langsam bis zu unserem heutigen Entwicklungsstand weiterbrachten. Während sich diese Ereignisse seit etwa 150 Jahren geradezu überschlagen, gibt es natürlich auch Epochen, die dazu wenig oder nichts beisteuerten, und dazu gehört vor allem das Mittelalter. Ausser einer eher zufällig gelungenen Pestprophylaxe durch Räucherungen und der sozial bedeutsamen Lepradiagnostik hatte es offenbar nur wenig zu bieten. Von aussen und unter dem Blickwinkel des Fortschritts betrachtet, sieht das Mittelalter vielleicht wirklich so aus, deshalb hat der Mediävist für diese Art der Darstellung ein gewisses Grundverständnis, auch wenn es weh tut, dass die Ergebnisse von rund hundert Jahren Forschungsgeschichte ignoriert und auch faktische Fehler nicht korrigiert werden. ${ }^{2}$ Gegen Weitergabe von Klischees in Lehrbüchern für Nicht-Fachleute und Anfänger ist der Spezialist machtlos und findet sich damit wohl auch ab (ausser dem Mittelalterkapitel dürfte der Abschnitt über Paracelsus darunter am meisten zu leiden haben); wenn aber ein angesehener Medizinhistoriker in einem wissenschaftlichen Aufsatz erst neulich wieder schreibt, dass «das Feld der mittelalterlichen Medizingeschichte so gut wie unbeackert» sei,$~^{3}$ so lässt das einem keine Ruhe. Alle Ergebnisse sind natürlich publiziert, aber gleichzeitig scheinen sie nicht rezipierbar zu sein: ein klassisches Kommunikationsproblem, bei dem die Botschaft des Senders beim Empfänger nicht ankommt.

Bevor wir nach den Gründen fragen, sind einige Vorbemerkungen nötig: Erstens gibt es in der Tat einen weissen Fleck in der Erforschung des Mittelalters, und zwar im Bereich der lateinischen Medizinliteratur, die den Schriftenkanon der Universitäten stellte. Obwohl Karl Sudhoffs Start in die professionell betriebene Medizingeschichte zweigleisig erfolgte, ist, von wenigen Ausnahmen abgesehen, ${ }^{4}$ die Arbeit nur notdürftig anhand alter Ausgaben und auf der Basis von Frühdrucken möglich. ${ }^{5}$ Über Text- und Überlieferungsgeschichte ist nur wenig bekannt, auch die Kommentare zu den Klassikern müssten noch aufgearbeitet werden. Wenn also z. B. die umfangreiche Handschriftensammlung der beiden gelehrten Nürnberger Ärzte Hartmann und Hermann Schedel trotz ihrer höchst attraktiven Ausstat- 
tung noch nicht erschlossen ist, ${ }^{6}$ so liegt das schlicht daran, dass die einfachste Grundlage der Arbeit fehlt, denn viele der lateinischen Texte der Kodizes sind beim heutigen Forschungsstand noch gar nicht in ihrem Traditionszusammenhang oder gar in ihrer Redaktionsstufe bestimmbar. Weshalb sich niemand so recht an die bedeutenden Werke heranwagt, ist klar: Mittellatein ist zwar nicht allzu schwierig, doch bedarf der Fachwortschatz einer gewissen Einarbeitung, zumal auch er nur unzureichend erfasst ist und eine Übersetzung oft gleichzeitig eine Interpretation sein muss, mit der beständig Neuland betreten wird. Ausserdem sind die meisten Handschriften schwer lesbar, da die Abbreviaturen nicht die üblichen sind und interindividuell noch dazu schwanken. Doch der Hauptgrund ist, dass jeder Bearbeiter sofort mit einer derartigen Fülle von Material von Parallelüberlieferungen konfrontiert wird, dass seine ganze Leistungskraft gleich für viele Jahre an einem einzigen Projekt gebunden wäre, und das kann sich im Zeitalter kurzzeitig befristeter Verträge und des Leitsatzes «publish or perish» so gut wie niemand mehr erlauben. Was käme auch heraus? Gemessen am nötigen Aufwand, ist wahrscheinlich der Erkenntnisgewinn einer textkritischen, überlieferungsgeschichtlichen und kommentierten Ausgabe von Avizennas «Canon» oder des «Antidotarium Nicolai» nicht sensationell genug, um Geldgeber und Gutachter über einen entsprechend langen Zeitraum hinweg bei der Stange zu halten.

Und damit sind wir bei der zweiten notwendigen Vorbemerkung angelangt, die uns bereits auf eine wichtige Voraussetzung für eine funktionierende Kommunikation führt, nämlich das Interesse des Empfängers. Gleichgültig, ob man es begrüsst oder bedauert (oder, wie gleich im folgenden, begründet), es bleibt ein Faktum, dass die Arbeit am mittelalterlichen Fachtext zur Zeit nicht en vogue ist. Die Medizingeschichte des deutschsprachigen Raums, die die Historie der grossen Namen satt hat, sucht internationalen Anschluss an verwandte Disziplinen und findet Ansprechpartner einerseits in der Wissenschafts- und anderseits in der Sozialgeschichte. Gerade letztere übt durch ihre völlig anderen Ansätze, die einen Paradigmenwechsel einläuteten, ${ }^{7}$ eine derart faszinierende Sogwirkung aus, dass dadurch nicht nur das Erkenntnisinteresse absorbiert wird, sondern dass der traditionelle, philologische Blickwinkel dagegen langweilig, ja anachronistisch aussieht. Trotz vielversprechender erster Ergebnisse ist auf diesen neuen Gebieten, die hierzulande relativ jung sind, gerade für das Mittelalter noch so viel Arbeit zu leisten, dass verständlich ist, wenn sich das Engagement auf diese Ziele richtet. Doch auch wenn Orientierung, Fragestellung 
und Methode sich mit guten Gründen verändert haben, so erscheint doch seltsam, dass das auf früheren Gebieten Erreichte ohne Echo bleibt.

Wovon hier die Rede sein soll, ist derjenige Sektor der mittelalterlichen Medizingeschichte, der mit Abstand am besten erforscht ist, ja der so gut erschlossen ist, dass ein abschliessendes Resümee in greifbare Nähe gerückt sein dürfte. ${ }^{8}$ Gleichzeitig ist es das Kapital, das nach aussen am wenigsten in Erscheinung tritt, also am «unbekanntesten» ist : die landessprachige Fachliteratur ist weder durch ihre hervorragendsten Vertreter noch in ihren Ausprägungsformen noch mit ihrem Inhalt präsent. Wir wollen daher untersuchen, woran es liegt, dass dieses umfangreiche, aber leicht zugängliche heilkundliche Schrifttum derart im Abseits steht.

\section{Das Fehlen des Rezeptionsraums}

Äusserliche Gründe spielen natürlich immer eine gewisse Rolle. Daher ist zunächst darauf hinzuweisen, dass die Wurzeln dieser Forschungsrichtung in der Germanistik der vierziger Jahre liegen. So ungewöhnlich und progressiv es damals war, als sich Gerhard Eis von der höfischen Literatur ab- und mittelalterlichen Fachtexten zuwandte, so sehr erweist es sich heute als hinderlich, dass die Verankerung in der Philologie und nicht in der Geschichte liegt, denn ein Literaturwissenschaftler geht anders mit Texten um als ein Historiker, für den schon das Wort «Quelle» eine ganz andere Bedeutung hat; Verständigungsschwierigkeiten sind also vorprogrammiert. Gleichzeitig erfolgte aber auch ein Wegdriften von der Altgermanistik, obwohl diese sich zunehmend für Fachliteratur zu interessieren begann, ${ }^{9}$ da die Medizingeschichte in anderem Umfeld organisiert ist; das wiederum behinderte den Austausch über neuere philologische Methoden. Das heisst: für Medizinhistoriker sind die Arbeiten der Eis-Schule zu sehr aufs Sprachliche ausgerichtet, und für Germanisten geraten sie so gut wie nicht ins Visier, da sie meist an für diese entlegener Stelle erscheinen. Historikern liegt wenig oder nichts an philologischer Kleinarbeit, wie an Textkritik und Gestaltwandel, an Überlieferungs- und Wirkungsgeschichte, an Kommentaren und Glossaren. Für Literaturwissenschaftler wiederum wirkt der Inhalt abschreckend : für medizinische Lehrbücher und den entsprechenden Fachwortschatz fühlen sie sich beim besten Willen nicht zuständig. Da also jeweils eine Hälfte der Untersuchungen auszublenden wäre, bleiben verdienstvolle Arbeiten als Ganzes unbeachtet. 


\section{Das Fehlen einer Gesamtdarstellung}

Bis heute fehlt eine Überblicksdarstellung der Ergebnisse, die dem Forschungsstand gerecht wird, ${ }^{10}$ und dieses Fehlen einer festen Diskussionsplattform scheint mir der Hauptgrund dafür zu sein, dass mögliche Rezipienten an der gesamten Richtung vorbeigehen. Details - und seien sie noch so attraktiv - finden nur Beachtung, wenn sie das Ganze illustrieren und beleuchten; wenn das Ganze aber nur aus heterogenen und primär unverbundenen Details besteht bzw. zu bestehen scheint, gibt es keinen Ansatz für eine fundierte Auseinandersetzung. Wie soll sich also ein Kollege aus anderen Richtungen informieren? Was findet ein Aussenstehender auf der Suche nach einem Einstieg?

Das Riesenœuvre Karl Sudhoffs besteht zum ganz überwiegenden Teil aus Kurzmitteilungen von Einzelfunden, die in wenigen Fällen nach Themengebieten locker geordnet sind. Weder Systematik noch Vollständigkeit waren Ziel des Autors; ihm lag daran, erst einmal möglichst viel aus der Fülle der Denkmäler bekanntzumachen. Sich darin zurechtzufinden, ist nicht nur ein mühseliges Unterfangen, sondern man kann auch noch sicher sein, dass am Ende nicht der aktuelle Wissensstand erreicht ist. Als Gerhard Eis die planmässige Erschliessung der mittelalterlichen Fachliteratur in Angriff nahm, stand die Medizin nicht allein im Zentrum seines Interesses. Die entsprechenden Kapitel in seinen beiden leicht zugänglichen Überblicksdarstellungen sind also überschaubar. Doch zu entnehmen ist ihnen nicht viel, da lediglich Namen von Autoren und Titel von Werken aneinandergereiht werden. In diesem merkwürdigen Vernachlässigen des Inhalts ist die EisSchule übrigens ihrem Lehrer treu geblieben: auch die Abschnitte in den Abrissen Peter Assions geben darüber keinen Aufschluss und präsentieren nur das Gerippe des Materials. Doch ausgerüstet mit diesen Hinweisen gelingt immerhin der Zugriff auf die wichtigste Quelle zur mittelalterlichen Medizinliteratur, nämlich auf die Neuauflage des «Verfasserlexikons». ${ }^{11}$ Die Artikel dort sind allerdings wesentlich zahlreicher und vielfältiger, da Vollständigkeit angestrebt wird, und sie spiegeln nun zwar den Stand der Forschung, legen aber den Schwerpunkt nicht auf den medizinhistorischen Wert eines Textes, sondern auf seine Text-, Überlieferungs- und Wirkungsgeschichte. Dennoch gewinnt der Leser auch einen Eindruck von Aufbau und Struktur. Die Zielrichtung geht jedoch eindeutig auf ein germanistisches Publikum. Einsicht in das Wesen der volkssprachigen Heilkunde wird der Historiker daher nicht erlangen (wenn er überhaupt ein Lexikon als Nach- 
schlagewerk akzeptiert), sondern nach kurzer Zeit von der Vielzahl der Traktate geradezu erschlagen werden, ohne dass er den berühmten «Wald hinter den Bäumen» erahnen könnte. Oder gibt es am Ende gar keinen «Wald», entziehen sich vielleicht die Fachtexte einem vereinheitlichenden Zugriff, der in ihre Buntheit und Fülle Systematik und Harmonie bringen würde? Die unzähligen kleinen Texte stecken in der Tat voller Widersprüche, so dass die ausgeprägten Skrupel vor einer Zusammenschau bis zu einem gewissen Grad verständlich erscheinen. ${ }^{12}$

\section{Die Problematik der Abgrenzung des Gegenstands}

Wie soll man medizinische Fachliteratur im Mittelalter definieren? Die Frage klingt töricht, da der Gegenstand nach heutigem Verständnis keine Erkenntnisschwierigkeiten bereitet. Befremdlich ist jedoch schon, dass Gerhard Eis sich bei der Abgrenzung der «Artesliteratur» (Fachprosa, Fachliteratur) auf ein Ausklammern des juristischen und religiösen Schrifttums festlegte, beides Textkorpora, die wesentlich grösser als das der «Fachliteratur» sind. Erst die Germanistik der letzten Jahre ist dazu vorgestossen, alle «nicht-belletristischen» Texte unter einem gemeinsamen Dach, dem der «Sachliteratur», zusammenzufassen. Die Arbeit am historischen Text hat ferner gezeigt, dass die an Hugo von St. Viktor orientierte Aufspaltung nach den artes liberales, mechanicae und magicae, die Eis vorschlug und von der die Forschungsrichtung ihren Namen hat (Artesforschung), nicht trägt. Zu den «eigentlich» medizinischen Texten gehören nämlich ganz selbstverständlich auch von den Freien Künsten die Astrologie (Planetenkinder- und Tierkreiszeichenlehren, Horoskope, Lunare, Kalender) und von den Verbotenen Künsten Magie und Mantik, ohne dass je im Mittelalter ein Zuordnungsproblem gesehen oder reflektiert worden wäre. Innerhalb der «Eigenkünste»(der Fachliteratur im engeren Sinne nach Eis) überschneidet sich die Heilkunde mit agricultura und venatio, nicht nur wegen der Nachbarschaft zur Veterinärmedizin, sondern auch wegen der Nähe zu Botanik (Kräuterbücher), Zoologie (Wunderdrogen) und Mineralogie (Materia medica, Steinbücher). Der Kontext erlaubt sogar einen Brückenschlag zu hauswirtschaftlichen Texten (lanificium), denn die heilkundlichen Rezepte sind nicht nur Kochbüchern benachbart, sondern stehen auch neben technologischen Anweisungen (Färbebüchlein). Fazit: Eine neue Darstellung zumindest der medizinischen Fachtexte müsste sich endgültig von der Eis'schen Abstraktion lösen 
und den zeitgenössischen Zusammenhang rekonstruieren, dabei also Fächerüberschneidungen in der Weise akzeptieren, wie sie aus den Handschriften ersichtlich sind. Erst dann kann man zu einer sowohl in sich schlüssigen als auch Aussenstehende überzeugenden Gliederung des Gegenstands gelangen. ${ }^{13}$

\section{Titel für Texte ohne Werkcharakter}

Der historische Befund ist auch noch auf einer anderen Diskursebene stärker zu berücksichtigen: Von der Vielzahl der mittlerweile etablierten Titel war bereits die Rede. Ihre Funktion lag und liegt in der Vereinfachung der Kommunikation, denn es sollten dadurch Parallelüberlieferungen unter einem Dach zusammengefasst werden. Mittlerweile hat diese Intention sich jedoch in ihr Gegenteil verkehrt. Angesichts der ungeheuren inzwischen erschlossenen Materialfülle wäre es praktischer, Handschriftensignaturen und Blattzahlen anzugeben, als vom «Deutschen Salernitanischen», ${ }^{4}$ «Kleinen niederdeutschen», ${ }^{15}$ «Darmstädter» ${ }^{16}$ usw. «Arzneibuch» zu sprechen. Überschneidungen, die aus unterschiedlichen Bearbeitungsstadien resultieren, würden dann sofort auffallen. Ausserdem wäre dann geklärt, was mit den häufig anzutreffenden Ortsnamen gemeint ist: der Herkunftsort eines Kodex (Schreibstube oder Bibliothek), eine zeitweilige, aber wichtige Zwischenstation oder der jetzige Aufbewahrungsort, vielleicht sogar der (vermutete) Entstehungsort eines Textes oder eines Textkorpus, die in der Handschrift überliefert sind. Gegen die Titelvergabe ist jedoch noch ein schwerer wiegendes Bedenken anzuführen: ${ }^{17}$ ein Titel suggeriert ein Werk mit Anfang und Ende, das einem bestimmten Verfasser zuzuschreiben ist (auch wenn wir ihn nicht kennen sollten). Er suggeriert ferner für Uneingeweihte ein gewisses, sogar relativ hohes Niveau. Beide Assoziationen führen in die Irre : bei weitem der grösste Anteil der landessprachigen medizinischen Texte des Mittelalters sieht ganz anders aus. Es handelt sich um ad hocKompilationen, die natürlich alle zu einem Thema irgendwie ähnlich aussehen, die aber als Individuen zu betrachten sind, von denen jedes aus anderem Quellenmaterial zusammengestückelt wurde. Dazu kommt, dass ihre Reihung innerhalb der Handschrift assoziativ ist; die Themengebiete gehen daher nahtlos ineinander über. Wo ein solcher «Text» beginnt und wo er aufhört, ist daher oft nur willkürlich festzumachen. Dass dieses historische Faktum die Kommunikation nicht gerade erleichtern wird, ist klar: 
Texte ohne Werkcharakter haben nicht nur keinen konstanten Inhalt, sondern verkehren unter Umständen sogar Aussagen in ihr Gegenteil, da ihre Schreiber in der Regel nicht mit dem Gegenstand vertraut sind, sondern sich über die Vielzahl der Vorlagen absichern. Eine gemeinsame Basis daraus zu konstruieren, wird schwierig sein. Dennoch scheint mir das Unternehmen zukunftsträchtig, statt Einzelbeispielen Gattungen vorzustellen (wie es im "Verfasserlexikon» teilweise schon versucht wird), ${ }^{18}$ auch wenn die Textsortenbestimmung ein weiteres schwieriges Problem ist. Nicht nur in der Belletristik, die gerade für die spätmittelalterliche Literatur noch keine überzeugende Gattungstheorie gefunden hat, sondern in erheblichem Umfang auch bzw. gerade in der Fachprosa ist mit einer «permanenten Mutation ${ }^{19}$ zu rechnen. Das bedeutet: Textarten definieren sich nicht nur nach Form und Inhalt, sondern auch nach ihrer Funktion im Kontext, die als Variable anzusehen ist.

Durch das Zurechtrücken der Textdignität vergibt sich die Artesforschung nichts, etwa in dem Sinne, dass die von ihr bearbeiteten und vorgestellten Traktate nichts wert wären. Im Gegenteil: sie macht klar, dass die Heilkunde keine Sonderrolle innerhalb der Sachliteratur spielt, für die die gleichen Beobachtungen gelten, und ordnet sich damit in den Gesamtzusammenhang der Spätmittelalterforschung ohne Brüche ein. Dem (Medizin)Historiker, der heute nicht mehr daran interessiert ist, möglichst früh eine möglichst spektakuläre Entdeckung möglichst an seiner Heimatuniversität zu dokumentieren, wird auf diese Weise eine beachtliche Materialmenge an die Hand gegeben, die die «einfache» Medizin spiegelt, die also aktuelle Begriffe wie «Gesundheits-» und «Krankheitsverhalten» («Coping») illustrieren kann, die den Hintergrund für Prozessakten und Leichenpredigten abgibt, die klärt, wie die Angaben der Todesursachen in den Kirchenbüchern zu verstehen sind, die die Kriterien der Lepraschau mit Inhalt füllt usw. Insbesondere ist auf das erweiterte Literaturverständnis der Mediävistik hinzuweisen, das praktisch bloss noch die Kriterien Schriftlichkeit und Geschichtlichkeit anwendet und lediglich noch die Urkunden ausklammert. ${ }^{20}$ Viele früher als «typisch» sozialgeschichtlich eingestuften Quellen sind damit gleichzeitig Gegenstand der Fach- bzw. Sachprosaforschung geworden. Die Titelvergabe erweist sich damit als weit mehr als nur eine Frage der Wissensorganisation oder als literaturtheoretisches Problem, sondern führte - ohne dies je beabsichtigt zu haben - zu einer Polarisierung in der Methodendiskussion, die durch die tägliche Arbeit in nichts gerechtfertigt ist. 


\section{Terminologische Probleme}

Was die Kommunikation ebenfalls erschwert, oft sogar verhindert, sind Verständigungsschwierigkeiten auf der simplen Ebene der Wortbedeutung. Bei einer jahrzehntelangen Forschungstradition bleibt es nicht aus, dass sich ein «Insider-Jargon» entwickelt, der für die Eingeweihten den Vorteil der raschen Übermittlung einer Fülle von Informationen anhand eines Stichworts hat; für die Darstellung nach aussen ist dieses in fast allen Sparten der Wissenschaften zu beobachtende Phänomen jedoch ungünstig, wenn nicht deletär. Sobald der allgemeinsprachliche Bedeutungsrahmen nämlich verlassen wird, sind Missverständnisse vorprogrammiert: auf diese bekannten Mechanismen wird schon der Medizinstudent im ersten Semester während seines Terminologie- oder Soziologiekurses aufmerksam gemacht. Die Fachsprache der Artesforschung nun hat für wesentliche und häufige Begriffe Sonderbedeutungen und -bezeichnungen kreiert, die die Gefahr von Fehlassoziationen in sich bergen. Zwei besonders eindrückliche und gebräuchliche Beispiele mögen ausreichen, um dies zu illustrieren.

Einfach scheint ein Wort wie «Aderlassbüchlein» zu sein, ${ }^{21}$ denn jeder weiss, was die beiden Komponenten meinen, aus denen das Wort zusammengesetzt ist. Doch erst die Kontrolle der einschlägigen Sekundärliteratur und das Nachlesen der Texte selber klärt den Sachverhalt: Nur in Ausnahmefällen handelt es sich um echte «Büchlein» im Sinne kleiner Kodizes (klein und handlich im Format, klein auch im Umfang). Sehr viel häufiger sind Abschnitte in grösseren Sammelhandschriften gemeint, die sich noch dazu keineswegs klar von ihrer Umgebung abgrenzen lassen (s. Punkt 4). Noch verblüffender ist, dass die Phlebotomie nur eine Klammer ist, die ganz unterschiedliche Traktate lose zusammenhält; die «Mitüberlieferung» deckt ein Spektrum ab, das von Harntraktaten bis zu einfacher Astrologie reicht ${ }^{22}$ und sogar regelrechte Rezeptare nicht ausschliesst. ${ }^{23}$

Unter einem Bedeutungswandel hat das Wort «Arzneibuch» zu leiden. ${ }^{24}$ Heute gehört es in den Sprachgebrauch der Apotheker, steht also für eine Pharmakopöe, und diese moderne Variante kommt selbstverständlich auch in historischem Kontext zum Tragen. Eine solche (Fehl)Assoziation war im übrigen auch der Grund, weshalb James Follans Erstausgabe von Ortolfs von Baierland Arzneibuch in der Schriftenreihe der Internationalen Gesellschaft für Geschichte der Pharmazie erschienen ist (Stuttgart 1963). An diesem medizinischen Lehrbuch zeigt sich, dass die am häufigsten benutzte Grundbedeutung des Wortes die mittelhochdeutsche ist («Buch von der 
Arznei» $=$ «Buch von der Heilkunde»). Während Ortolf ein zusammenhängendes Werk verfasst hat (eben das, was wir unter einem «Buch» verstehen), hat sich aber noch eine ganz andere Verwendung eingebürgert: es werden nämlich Sammelhandschriften überwiegend medizinischen, aber auch bunt vermischten Inhaltes, bei dem die Heilkunde nur eine Nebenrolle spielt («Bücher von Mensch, Tier und Garten»), als «Arzneibücher» bezeichnet («Darmstädter», «Kasseler», «Stockholmer», «Utrechter Arzneibuch»). Diese letztgenannte Sonderform des «Arzneibuchs» sprengt natürlich den Rahmen der Grundbedeutung in gewisser Weise. Um dieses Unbehagen zum Ausdruck zu bringen, wird eine Reihe konkurrierender Bezeichnungen angewandt : vermischte Sammelkodizes heissen daher zusätzlich sowohl «Kompendien» als auch «Enzyklopädien» ${ }^{25}$ und nehmen damit zwei weitere Gattungsbezeichnungen für sich in Anspruch, die nach allgemeinem Verständnis völlig andere, ja sogar gegensätzliche Assoziationen hervorrufen («Lucidarius», «Buch der Natur»), auch wenn natürlich einzelne Definitionskriterien zutreffend sind: im Wort «Kompendium» wird das Zusammenfassen von zahlreichen Einzelinformationen auf engstem Raum betont, im Wort «Enzyklopädie» schwingt die umfassende Vielfalt des Mitgeteilten mit. Beides steht jedoch für ein einheitliches «Werk», nicht für eine lockere Aneinanderreihung von Kompilaten verschiedener Thematik.

Es ist wenig sinnvoll, weiter ins Detail zu gehen. Festzuhalten bleibt, dass die einfachste Basis für ein Gelingen von Kommunikation, nämlich die gemeinsame Sprache, erst noch zu finden ist. Termini müssen eindeutig sein, d.h. sie dürfen nur einen genau definierten Gegenstand bezeichnen, und es sollte dafür keine Konkurrenzbenennung geben. Dass eine Gesamtdarstellung der Forschungsergebnisse erst einmal mit einer Art Lexikon des Sprachgebrauchs anfangen muss, macht ein solches Unternehmen natürlich nicht besonders attraktiv, zumal man sich von liebgewonnenen und längst nicht mehr reflektierten Wörtern verabschieden müsste. Ohne diese mühsame Knochenarbeit gibt es jedoch keine Basis für gelungene Wissensvermittlung.

\section{Selbstbeschränkung}

Ein Wort gibt es in der Fachprosaforschung, das wie kein zweites Unbehagen hervorruft, nämlich der «Gebrauchstext». Dementsprechend trennt die Germanistik auch «Bildungswissen» vom «Handlungswissen» ab. Jeder 
Sozialhistoriker wird sofort mit dem berechtigten Argument zur Stelle sein, die Fachprosaforschung könne nicht für sich in Anspruch nehmen, «die» Medizin des Mittelalters zu erfassen und darzustellen, sie beschreibe vielmehr nur die medizinischen Texte, von denen erst zu beweisen wäre, dass sie gleichsam «Gebrauchsanleitungen» für die Praxis sind. ${ }^{26}$ Der Einwand zwingt in der Tat zu Selbstreflexion und Selbstbescheidung, wobei diese jedoch durchaus mit erheblichem Erkenntnisgewinn für alle Seiten einhergehen. Die Frage nach dem «Sitz im Leben» von Texten gehört zum Repertoire jedes Literaturwissenschaftlers und muss auch die Artesforschung beschäftigen. Spiegelt ihr Gegenstand also die professionelle Medizin in Händen von Personen ohne Lateinkenntnis, die Laienmedizin oder gar die sogenannte Volksmedizin? Was «ist» mittelalterliche Chirurgie? Der Text des berühmten Wundarztes? Seine Umsetzung? Wie soll ich diese aber beweisen, wenn ich nur den Text betrachte? Derartige Fragen differenziert für den Einzelfall zu beantworten, sich die Grenzen einer Methode und des Forschungsstandes bewusst zu machen sowie Ergebnisse anderer Ansätze einzubeziehen, führt sicher weiter als ein allzu forsches Reklamieren absoluter Aussagesicherheit bei fragwürdigem Fundament. Jede historische Quelle ist in ihrer Auswertbarkeit limitiert, keine bietet die ganze Wirklichkeit, sondern nur einen Ausschnitt. Diesen aber voll auszuschöpfen, bedeutet, einen einsatzfähigen Baustein in die Rekonstruktion der geschichtlichen «Realität» zu liefern. Mein Plädoyer ermuntert also ebenso zu Selbstbewusstsein wie zu Selbstkritik: erst wenn der eigene Beitrag zur Medizingeschichtsschreibung wirklich definiert ist, sollte sich die Diskussion um seinen Wert entfalten. 


\section{Anmerkungen}

1 Darüber Bernhard Schnell: Die deutsche Medizinliteratur des Mittelalters in den «Geschichten der Medizin». Vortrag, gehalten auf der Jahrestagung der Würzburger Medizinhistorischen Gesellschaft 1993, erscheint 1994 in den Würzburger medizinhistorischen Mitteilungen. Vgl. auch Anm.2.

2 Um nur ein Beispiel zu nennen: Das Ortolf von Baierland zugeschriebene Frauenbüchlein aus dem späten 15. Jh. wird noch immer für echt gehalten, obwohl längst bekannt ist, dass der berühmte Würzburger Arzt im 13.Jh. lebte. Vgl. z. B. Dieter Jetter: Geschichte der Medizin, Stuttgart u. New York 1992, S.218; vgl. dagegen Gundolf Keil: Ps.-Ortolfisches Frauenbüchlein. In: Verfasserlexikon (wie Anm. 11) 7. Bd., Sp. 82-84.

3 Richard Toellner: Der Körper des Menschen in der philosophischen und theologischen Anthropologie des Spätmittelalters und der frühen Neuzeit. In: Gepeinigt, begehrt, vergessen. Symbolik und Sozialbezug des Körpers im späten Mittelalter und in der frühen Neuzeit. München 1992, S.131-146, Zitat S. 131.

4 Die Werke Arnalds von Villanova beispielsweise werden unter der Leitung von Luis Garcia Ballester durch die Universität Barcelona erschlossen.

5 Sogar für die bedeutendsten Werke sind wir auf Faksimile-Nachdrucke von Inkunabeln angewiesen: Liber Canonis Auicenne reuisus et ob omni errore mendaque purgatus summaque cum diligentia impressus, Venedig: Paganinus de Paganinis, 1507, Neudr. Hildesheim 1964; Dietlinde Goltz: Mittelalterliche Pharmazie und Medizin. Dargestellt an Geschichte und Inhalt des Antidotarium Nicolai. Mit einem Nachdruck der Druckfassung von 1471. Stuttgart 1976 (Veröffentlichungen der Internationalen Gesellschaft für Geschichte der Pharmazie e. V., Neue Folge, 44).

6 So Bernhard Schnell auf der Jahresversammlung 1993 des Arbeitskreises Alte Medizin in Mainz.

7 Robert Jütte: Sozialgeschichte der Medizin. Inhalte - Methoden - Ziele. In : Medizin, Gesellschaft und Geschichte 9 (1990), S. 149-164; Francisca Loetz: Histoire des mentalités und Medizingeschichte. Wege zu einer Sozialgeschichte der Medizin. Medizinhistorisches Journal 27 (1992), S. 272-291.

8 Johannes G. Mayer: Wissenschaftsbericht des Instituts für Geschichte der Medizin in Würzburg im Rahmen des Sonderforschungsbereichs 226. Nachrichtenblatt der deutschen Gesellschaft für Geschichte der Medizin, Naturwissenschaft und Technik 43 (1993), S. 97101 .

9 So gab es z. B. von 1984 bis 1992 in Würzburg und Eichstätt einen DFG-Sonderforschungsbereich (226) über «Wissensorganisierende und wissensvermittelnde Literatur des Mittelalters»; die entsprechenden Arbeiten erscheinen in der Reihe «Wissensliteratur im Mittelalter». Dabei wurde neben medizinischem auch religiöses, geschichtliches, geographisches und enzyklopädisches Wissen untersucht. Typisch ist auch die Reihe «Text und Textgeschichte», in der z. B. die «Rechtssumme» Bruder Bertholds, der «Vocabularius Ex quo» und die «Elsässische Legenda aurea» erschienen sind. Die Universität Münster bietet ein Graduiertenkolleg «Schriftkultur und Gesellschaft im Mittelalter» an.

10 Gerhard Eis: Mittelalterliche Fachprosa der Artes. In: Deutsche Philologie im Aufriss. Hrsg. v. Wolfgang Stammler. 2. Bd. 2. Aufl. Berlin 1960, Sp. 1103-1215; ders. : Mittelalter- 
liche Fachliteratur. 2. Aufl. Stuttgart 1967 (Sammlung Metzler, 14); auf dieser Basis auch Peter Assion: Altdeutsche Fachliteratur. Berlin 1973 (Grundlagen der Germanistik, 13). Auf dem Forschungsstand der siebziger Jahre bleibt auch, da sich das Erscheinen des Bandes verzögerte: Peter Assion: Fachliteratur. In: Die deutsche Literatur im späten Mittelalter, 1250-1370. 2. Teil : Reimpaargedichte, Drama, Prosa. Hrsg. v. Ingeborg Glier. München 1987 (Geschichte der deutschen Literatur von den Anfängen bis zur Gegenwart, III.2), S. 371-395.

11 Die deutsche Literatur des Mittelalters. Verfasserlexikon. 2. Aufl. Hrsg. v. Kurt Ruh u. a. Berlin u. New York 1978 ff., bisher 8 Bde. Vgl. auch Anm.1.

12 Gundolf Keil: Der medizinische Kurztraktat in der deutschen Literatur des Mittelalters. In: Beiträge zur Überlieferung und Beschreibung deutscher Texte des Mittelalters. Referate der 8. Arbeitstagung österreichischer Handschriften-Bearbeiter vom 25.-28.11.1981 in Rief bei Salzburg. Hrsg. v. Ingo Reiffenstein. Göppingen 1983 (Göppinger Arbeiten zur Germanistik, 402), S.41-114. Keil spricht dort abschliessend von «widerspenstigen kleinen Texten» (S.114).

13 Ortrun Riha: Das systematologische Defizit der Artesforschung. Überlegungen zur mittelalterlichen deutschen Fachliteratur. Archiv für das Studium der neueren Sprachen und Literaturen 144 (1992), S. 255-276.

14 Gundolf Keil: Deutsches salernitanisches Arzneibuch. In: Verfasserlexikon (wie Anm. 11). 2. Bd., Sp. 69-71.

15 Agi Lindgren: «Kleines niederdeutsches Arzneibuch». In: Verfasserlexikon. 4.Bd., Sp. 1199 f.

16 Christian Tenner u. Gundolf Keil: Das «Darmstädter Arzneibuch». Randnotizen zu einer oberrheinisehen Sammelhandschrift der Zeitenwende. Bibliothek und Wissenschaft 18 (1984), S. 85-235.

17 Ortrun Riha: Wissensorganisation in medizinischen Sammelhandschriften. Klassifikationskriterien und Kombinationsprinzipien bei Texten ohne Werkcharakter. (Med. Habil.schr. Würzburg 1990) Wiesbaden 1992 (Wissensliteratur im Mittelalter, 9).

18 Z.B. Gundolf Keil u. Friedrich Lenhardt: Lepraschau-Texte. In: Verfasserlexikon. 5. Bd., Sp. 723-726.

19 Jörg-Ulrich Fechner : Permanente Mutation-Betrachtungen zu einer «offenen» Gattungspoetik. In: Die Gattungen in der Vergleichenden Literaturwissenschaft. Hrsg. v. Horst Rüdiger. Berlin u. New York 1974 (Komparatist. Stud. 4), S.1-31.

20 Kurt Ruh: Überlieferungsgeschichte mittelalterlicher Texte als methodischer Ansatz zu einer erweiterten Konzeption von Literaturgeschichte. In: Überlieferungsgeschichtliche Prosaforschung. Beiträge der Würzburger Forschergruppe zur Methode und Auswertung. Hrsg. v. Kurt Ruh. Tübingen 1985 (Text und Textgeschichte, 19), S. 262-272. Somit gehört beispielsweise auch der Text, den Robert Jütte in seiner Habilitationsschrift vorstellt und auswertet, zur «Literatur»: Ärzte, Heiler und Patienten. Medizinischer Alltag in der frühen Neuzeit. München u.ö 1991.

21 Ortrun Riha: Der Aderlass in der mittelalterlichen Medizin. Medizin, Gesellschaft und Geschichte 8 (1989), S. 93-118.

22 Hans Habernickel: Der Aderlassabschnitt des Codex Palatinus Germanicus 558. Quellenkritische und sprachliche Untersuchung zu einem bairischen Aderlassbüchlein des Spätmittelalters. Germanist. Doktoralabhandlung Nimwegen 1976 (masch.). 
23 Gerhard Eis u. Wolfram Schmitt: Das Asanger Aderlass- und Rezeptbüchlein (1516-1531). Stuttgart 1967 (Veröffentlichungen der Internationalen Gesellschaft für Geschichte der Pharmazie e. V., Neue Folge, 31); Gerrit Bauer: Das «Haager Aderlassbüchlein» (Studien zum ärztlichen Vademecum des Spätmittelalters, 1). (med. Diss. Würzburg) Pattensen 1978 (Würzburger medizinhistorische Forschungen, 14).

24 Riha: Wissensorganisation (wie Anm. 17), S. 7-18.

25 Volker Zimmermann: Die Heilkunde in spätmittelalterlichen Handschriftenenzyklopädien. In: Sudhoffs Archiv 67 (1983), S. 39-49; ders. : Rezeption und Rolle der Heilkunde in landessprachigen handschriftlichen Kompendien des Spätmittelalters. (med. Habil.schr. Würzburg 1982) Stuttgart 1986 (Ars medica, IV.2).

26 Von germanistischer Seite wurden schon länger Bedenken geäussert: Hugo Kuhn: Entwürfe zu einer Literatursystematik des Spätmittelalters. Tübingen 1980, S. 90.

Prof. Dr. med. Dr. phil. Ortrun Riha

Institut für Medizin- und Wissenschaftsgeschichte

Königstrasse 42

D-23552 Lübeck 